\title{
The Effects of Zinc Oxide (ZnO) Quantum Dots (QDs) Embedment on the Physicochemical Properties and Photocatalytic Activity of Titanium Dioxide $\left(\mathrm{TiO}_{2}\right)$ Nanoparticles
}

\author{
Anwar Iqbal, ${ }^{1 *}$ Usman Saidu, ${ }^{1}$ Farook Adam, ${ }^{1}$ Srimala Sreekantan, ${ }^{2}$ \\ Normawati Jasni, ${ }^{1}$ and Mohammad Norazmi Ahmad ${ }^{3}$ \\ ${ }^{1}$ School of Chemical Sciences, Universiti Sains Malaysia, 11800 Pulau Pinang, Malaysia \\ ${ }^{2}$ School of Materials and Mineral Resources Engineering, Universiti Sains Malaysia, \\ Engineering Campus, 14300 Nibong Tebal, Seberang Perai Selatan, \\ Pulau Pinang, Malaysia \\ ${ }^{3}$ Experimental and Theoretical Research Lab, Department of Chemistry, Kulliyyah of \\ Science, International Islamic University Malaysia, 25200 Kuantan, Pahang, Malaysia \\ *Corresponding author: anwariqbal@usm.my
}

Published online: 25 August 2021

To cite this article: Iqbal, A. et al. (2021). The effects of zinc oxide ( $\mathrm{ZnO}$ ) quantum dots (QDs) embedment on the physicochemical properties and photocatalytic activity of titanium dioxide $\left(\mathrm{TiO}_{2}\right)$ nanoparticles. J. Phys. Sci., 32(2), 71-85. https://doi.org/ 10.21315/jps2021.32.2.6

To link to this article: https://doi.org/10.21315/jps2021.32.2.6

\begin{abstract}
In this study, a detailed investigation on the effect of zinc oxide ( $\mathrm{ZnO})$ quantum dots (QDs) embedment on the physicochemical properties of anatase titanium dioxide $\left(\mathrm{TiO}_{2}\right)$ was conducted. The highly porous nanocomposite labelled as ZQT was prepared via the sol-gel assisted hydrothermal method. The powder X-ray diffraction (XRD) analysis indicates that the average crystallite size of the $\mathrm{ZnO} Q \mathrm{QD}$, anatase $\mathrm{TiO}_{2}$ $\left(\mathrm{TiO}_{2} \mathrm{NPs}\right.$ ) and $\mathrm{ZQT}$ were $4.45 \mathrm{~nm}, 9.22 \mathrm{~nm}$ and $11.38 \mathrm{~nm}$, respectively. Photoluminescent (PL) analysis detected the presence of defects related to $\mathrm{TiO}_{2}$, oxygen vacancies and quantum confinement effect (QCE) of the ZnO QDs in ZQT. These features enhanced the photodegradation of tetracycline (TC) under 48 watt of fluorescent light irradiation when ZQT (98.0\%) was used compared to $\mathrm{TiO}_{2} \mathrm{NPs}(32.4 \%)$ and $\mathrm{ZnO}$ QDs (68.8\%). The photodegradation activity was driven by $\mathrm{O}_{2}^{\bullet-}$ followed by ${ }^{\bullet} \mathrm{OH}$ and $h^{+}$.
\end{abstract}

Keywords: $\mathrm{ZnO}, \mathrm{TiO}_{2}$, quantum dots, photocatalysis, tetracycline 


\section{INTRODUCTION}

Many research interests focused on the advanced oxidation processes (AOPs) to eliminate antibiotics pollutants since they are effective compared to traditional wastewater treatment techniques. ${ }^{1}$ The AOPs are the techniques involving the generation of highly reactive hydroxyl radical $\left({ }^{\circ} \mathrm{OH}\right)$ to destroy a wide range of organic pollutants. ${ }^{2}$ Among various AOPs, heterogeneous photocatalysis is regarded as the most efficient technique for degrading organic contaminants into innocuous compounds. ${ }^{2}$

Semiconductors incorporated with quantum dots (QDs) are a new class of photocatalysts that have drawn extensive interest for their quantum confinement effect (QCE), high extension coefficient and larger surface-to-volume ratio. The QCE leads to the bandgap widening, resulting in the generation of energetic photoelectrons required for oxygen reduction. ${ }^{3}$ The QDs/semiconductor nanocomposite have been developed using various methods. ${ }^{4-7}$ However, most of these methods required an ageing time of more than $24 \mathrm{~h}$ and higher temperatures $\left(200^{\circ} \mathrm{C}\right.$ to $\left.400^{\circ} \mathrm{C}\right)$. It is also difficult to control the coverage and the sizes of the QDs. ${ }^{8}$

In this work, a zinc oxide $(\mathrm{ZnO}) \mathrm{QDs} /$ titanium dioxide $\left(\mathrm{TiO}_{2}\right)$ nanocomposite was synthesised using a sol-gel assisted hydrothermal method. The sol-gel method allows the nanocomposite to be prepared at a mild temperature and allows better control of the QDs size and coverage. The obtained nanocomposite showed higher efficiency than the pristine $\mathrm{TiO}_{2}$ and $\mathrm{ZnO}$ QDs in the photodegradation of tetracycline (TC) antibiotic.

\section{EXPERIMENTAL}

\subsection{Synthesis of Zinc Oxide Quantum Dots (ZnO QDs)}

The synthesis of $\mathrm{ZnO}$ QDs was carried out according to the method reported by Mahjoub et al. with some modifications. ${ }^{9}$ A $50.0 \mathrm{ml}$ solution of zinc acetate $(0.1 \mathrm{M})$ was prepared by dissolving $4.4 \mathrm{~g}$ of zinc acetate dihydrate (Sigma Aldrich, $\geq 98 \%$, Selangor, Malaysia) in $200 \mathrm{ml}$ of methanol (QReC) under vigorous stirring. The zinc acetate solution $\mathrm{pH}$ was reduced to $\mathrm{pH} 14$ by adding $\mathrm{KOH}(\mathrm{QReC})(1 \mathrm{M})$ solution drop by drop to obtain the ZnO QDs. The mixture was stirred for $1 \mathrm{~h}$ using a magnetic stirrer. To control the particle size, tetraethylorthosilicate (TEOS, Acros Organics, 98\%, Fair Lawn, NJ) solution $(0.25 \mathrm{ml})$ was added, followed by distilled water $(0.5 \mathrm{ml})$ to initiate the sol-gel 
reaction of silica on the surface of the $\mathrm{ZnO}$ QDs. The $\mathrm{ZnO}$ QDs was obtained by centrifugation and washed three times with methanol and distilled water to remove unreacted reactants. The obtained $\mathrm{ZnO}$ QDs was dried in the oven at $100^{\circ} \mathrm{C}$ for $12 \mathrm{~h}$.

\subsection{Synthesis of $\mathrm{ZnO}$ QDs/ $/ \mathrm{TiO}_{2}$ Nanocomposite}

Titanium(IV) tetraisopropoxide (TTIP, Across Organic, > 98\%, Fair Lawn, NJ), solution $(5.90 \mathrm{ml})$ was added into $200 \mathrm{ml}$ of an aqueous solution containing $2.0 \mathrm{~g}$ of soluble starch (Fisher Scientific, Leichestershire, UK). The solution was stirred for $10 \mathrm{~min}$ at $65^{\circ} \mathrm{C}$. The solution's $\mathrm{pH}$ was adjusted to $\mathrm{pH} 9$ by adding the ammonium hydroxide solution dropwise. The $\mathrm{ZnO}$ QDs $(0.81 \mathrm{~g})$ was added to the $\mathrm{TiO}_{2}$ sol and stirred for another $1 \mathrm{~h}$ at $85^{\circ} \mathrm{C}$. Finally, the mixture was centrifuged, and the obtained product was washed four times with ethanol $(\mathrm{QReC}$ chemical, $>99.7 \%$ ) and distilled water. It was then dried at $100^{\circ} \mathrm{C}$ for $12 \mathrm{~h}$ and calcined at $500^{\circ} \mathrm{C}$ for $2 \mathrm{~h}$ with a heating rate of $3^{\circ} \mathrm{C} \mathrm{min}^{-1}$. The obtained $\mathrm{ZnO}$ QDs/ $\mathrm{TiO}_{2}$ nanocomposite was labelled as ZQT. For comparison, pristine $\mathrm{TiO}_{2} \mathrm{NPs}$ was synthesised similarly but without ZnO QDs.

\subsection{Characterisation $\mathrm{ZnO}$ QDs/ $/ \mathrm{TiO}_{2}$ Nanocomposite}

The crystalline phase and crystallite size of the synthesised samples were investigated using powder X-ray diffraction (XRD) techniques. The XRD patterns were recorded on a BRUKER AXS D8, Germany advanced X-ray diffractometer with $\mathrm{Cu}-\mathrm{K} \alpha$ radiation $(\lambda=0.15478 \mathrm{~nm})$. The $2 \theta$ range was set between $20^{\circ}$ to $80^{\circ}$. The field emission scanning electron microscope and energy dispersive X-ray (FESEM/EDX) analysis was used to determine the morphology and the elemental composition of the samples, employing Leo Supra 50Vp FESEM, Netherland. High-resolution transmission electron microscopy (HRTEM) was used for the texture analysis, employing HRTEM $200 \mathrm{kV}$ with Field Emission, TECNAI G2 20 S-TWIN, FEI, resolution below $100 \mathrm{~nm}$, USA). The nitrogen adsorption-desorption (NAD) analysis (Micromeritic ASAP 2020 Surface Adsorption Porosimeter [SAP], USA) was used to estimate the surface area and the porosity of the synthesized samples. The nanocomposites optical properties were measured using the solidstate UV/Vis diffuse reflectance spectroscopy (PerkinElmer Lambda 35 UV/VIS Spectrometer, USA) method by scanning the reflectance of the samples in the range of $200 \mathrm{~nm}$ to $800 \mathrm{~nm}$. Photoluminescence (PL) spectra were measured at room temperature using PerkinElmer LS 55, USA fluorescence spectrophotometer at an excitation wavelength of $325 \mathrm{~nm}$. 


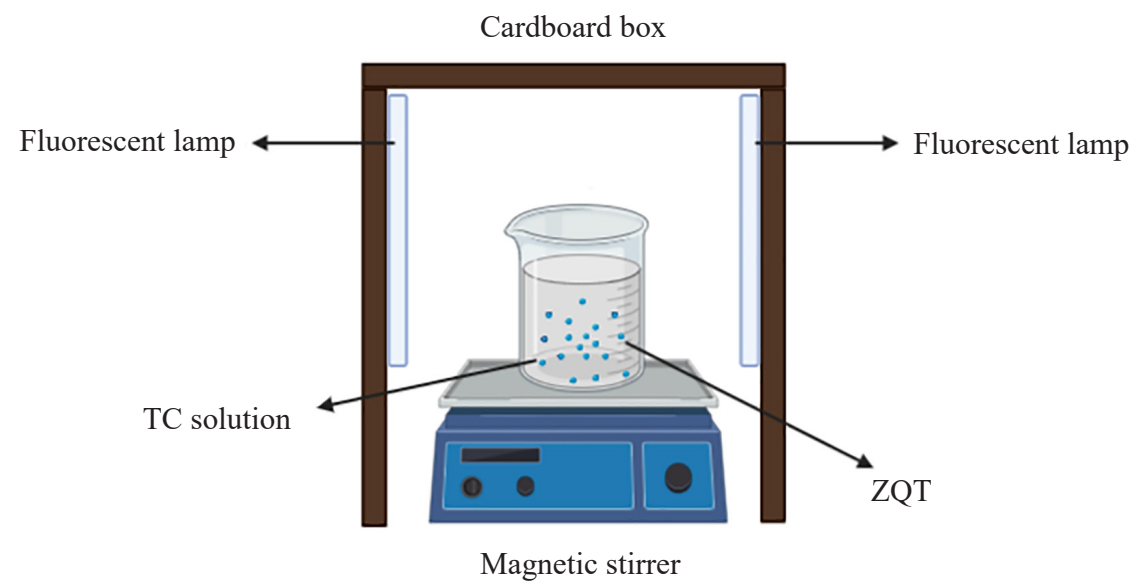

Figure 1: Homemade reactor used in the photodegradation of tetracycline.

\subsection{Photocatalytic Activity}

The photocatalytic performance of the nanocomposite was investigated by monitoring the photodegradation of TC in an aqueous solution using a homemade reactor (Figure 1) equipped with two fluorescent lights $(48 \mathrm{~W})$. The light intensity was determined to be $104.4 \mathrm{~W} / \mathrm{m}^{2}$, whereas the residual UV leakage of the visible light was detected to be $0.40 \mathrm{~W} / \mathrm{m}^{2}$. The measurement was carried out using Dual-Input Data Logging Radiometer (Model PMA2100, Pennsylvania, USA) equipped with visible, UVA + UVB detectors. In each experiment, $50 \mathrm{mg}$ of the photocatalyst was dispersed in $200 \mathrm{ml}$ of TC solution $\left(20 \mathrm{mg} \mathrm{l}^{-1}\right)$. Before the light irradiation, the suspension was magnetically stirred in the dark for 30 min to reach adsorption-desorption equilibrium. An aliquot from the suspension was then collected at regular time intervals and filtered using a $0.22 \mu \mathrm{m}$ membrane filter. The change in TC concentration was determined using a UV-vis spectrophotometer (UV2600, Shimadzu, Japan). The degradation percentage of the TC was calculated using the following equation:

$$
R(\%)=1-\frac{C_{t}}{C_{0}}
$$

Where $C_{0}$ represent the concentration before light irradiation, $C_{t}$ is the concentration at a determined reaction time, and $R$ is the percentage of TC photodegradation. The determination of the residual TC concentration was determined using the maximum absorbance at $358 \mathrm{~nm}$. 


\section{RESULTS AND DISCUSSION}

\subsection{XRD Analysis}

The XRD patterns of the materials are presented in Figure 2(a). The diffractogram of $\mathrm{TiO}_{2} \mathrm{NPs}$ shows the diffraction peaks of $2 \theta$ at $25.3^{\circ}, 37.8^{\circ}, 48.1^{\circ}, 54.1^{\circ}, 55.1^{\circ}$, $62.7^{\circ}, 68.7^{\circ}, 70.3^{\circ}$ and $75.2^{\circ}$ equivalent to the (101), (004), (200), (105), (211), (204), (116), (220), (215) and (224) planes.

These planes refer to the tetragonal structure of the anatase $\mathrm{TiO}_{2}$ phase (JCPDS 00-021-1272). The narrow and pointed diffraction peaks confirmed the high crystallinity of the anatase $\mathrm{TiO}_{2}$ phase. ${ }^{10}$

The XRD patterns of the as-synthesised ZnO QDs show broad diffraction peaks at $2 \theta$ values of $31.8^{\circ}, 34.5^{\circ}, 36.3^{\circ}, 47.6^{\circ}, 56.6^{\circ}, 63.0^{\circ}$ and $66.4^{\circ}$ corresponding to the crystal planes (100), (002), (101), (102), (110), (103) and (200) of hexagonal wurtzite phase of $\mathrm{ZnO}$ (JCPDS 36-1451). As can be seen from the XRD patterns of $\mathrm{ZQT}$, the crystalline planes indexed to the anatase $\mathrm{TiO}_{2}$ broadened when the $\mathrm{ZnO}$ QDs was incorporated into the crystal lattice of the anatase $\mathrm{TiO}_{2}$. The broadness of the XRD peaks indicates the formation of smaller size $\mathrm{ZnO}$ QDs crystallites. ${ }^{11}$ The average crystallite sizes as measured using the Debye-Scherrer equation (Equation 2) are $11.38 \mathrm{~nm}, 4.45 \mathrm{~nm}$, and $9.22 \mathrm{~nm}$ for the $\mathrm{TiO}_{2} \mathrm{NPs}, \mathrm{ZnO}$ QDs and ZQT, respectively.

$$
D=\frac{k \lambda}{\beta \cos \theta}
$$

Where $D$ is the crystallite size, $\lambda$ is the wavelength equal to $0.154 \mathrm{~nm}, k$ is a constant taken as $0.94, \beta$ is the full widths at half-maximum intensity (FWHM), and $\theta$ is the Bragg angle of the actual peak.

Diffraction peaks related to $\mathrm{ZnO}$ QDs were not observed in the diffractogram of ZQT. As the crystal framework of anatase was being built, it will induce strains to the crystal framework of the $\mathrm{ZnO}$ QDs. The strains may have caused the $\mathrm{ZnO}$ QDs to lose their crystallinity. ${ }^{12}$ As a result, crystalline peaks related to ZnO QDs were not detected. A lower concentration of $\mathrm{ZnO}$ QDs can also cause the absence of diffraction peaks. ${ }^{13}$ 

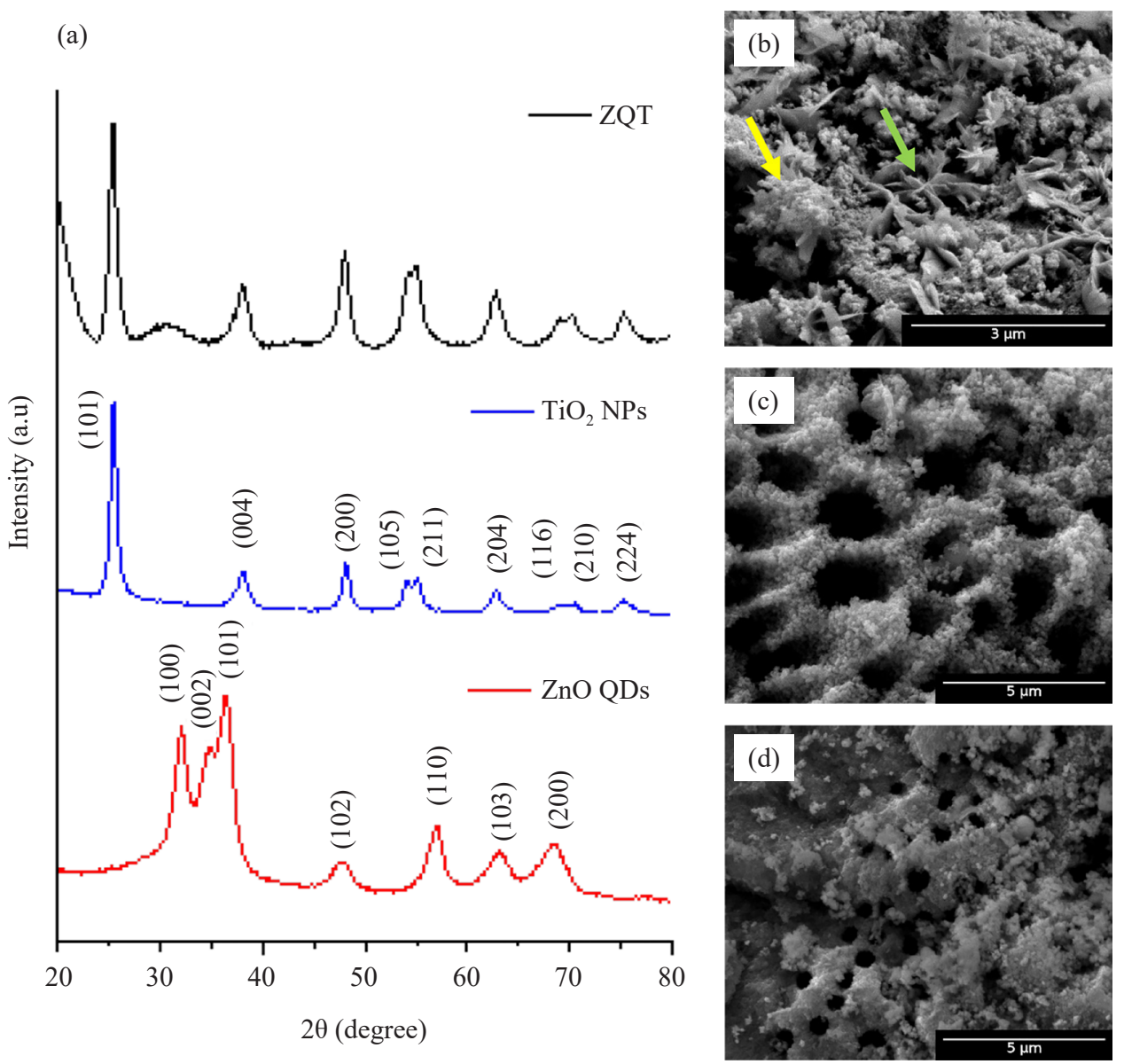

Figure 2: (a) XRD patterns of $\mathrm{ZQT}, \mathrm{TiO}_{2} \mathrm{NPs}$, and $\mathrm{ZnO}$ QDs, SEM images of (b) $\mathrm{ZnO}$ QDs, (c) $\mathrm{TiO}_{2}$ NPs, and (d) ZQT.

\subsection{Morphology Study}

The FESEM images of ZnO QDs are shown in Figure 2(b). The ZnO QDs was observed to contain irregularly spherical shaped particles (shown using a yellow arrow) and nanoflakes (shown using a green arrow). The formation of nanoflakes could be due to the rapid deposition of the $\mathrm{ZnO}$ QDs on top of the spherically shaped particles and begin growing upwards. The $\mathrm{ZnO}$ QDs can be attracted to each other due to electrostatic attraction. 
Starch was added as a template to create pores on the surface of the anatase $\mathrm{TiO}_{2}$. In hot water, the starch molecules will swell as a result of weakened hydrogen bonds. Due to the presence of Van der Waals forces, the $\mathrm{TiO}_{2}$ sols will be driven to be adsorbed around the starch molecules. The starch granules removed during the calcination process, leaving behind pores, as shown in Figure 2(c). ${ }^{14}$ The average pore diameter was measured to be $1.67 \mu \mathrm{m}$. The added $\mathrm{ZnO}$ QDs and $\mathrm{TiO}_{2} \mathrm{NPs}$ reacted to form a new composite layer and with an average pore diameter of $0.51 \mu \mathrm{m}$ (Figure 2[d]).

\subsection{Optical Absorption Property}

The UV-vis absorption spectra of $\mathrm{TiO}_{2} \mathrm{NPs}$ and ZQT are presented in Figure 3(a). The ZnO QDs was observed to have the lowest optical edge due to the quantum confinement effect (QCE). As the size of the increases, the optical band edge shifted to a higher wavelength. All the nanocomposites indicate good absorptivity in the UV region. The bandgap energies of the synthesised samples were calculated using Tauc's equation as follows:

$$
\alpha h v=\mathrm{A}(h v-E g)^{n / 2}
$$

Where $\alpha$ is the absorption coefficient, $h$ is Planck's constant, $v$ is the light frequency, $A$ is the proportionality constant, and $E g$ is the bandgap energy. The value of $n$ depends on the type of optical transition in the semiconductor, whether direct $(n=1)$ or indirect $(n=4)$ transition. The bandgap energies of the nanocomposites were estimated from the plot of $(\alpha h v)^{1 / 2}$ versus $h v$ as shown in the inset of Figure 3(a). The values were found to be $3.10 \mathrm{eV}, 3.50 \mathrm{eV}$ and $3.42 \mathrm{eV}$ for $\mathrm{TiO}_{2} \mathrm{NPs}, \mathrm{ZnO}$ QDs and ZQT, respectively. The Mulliken electronegativity theory was used to calculate the potentials of the valence band $(V B)$ and the conduction band $(C B)$ edges:

$$
\begin{aligned}
& E_{V B}=X-E^{e}+0.5 E g \\
& E_{C B}=E_{V B}-E g
\end{aligned}
$$

The $E_{V B}$ is the valence band edge potential, $E_{C B}$ is the conduction band potential, $E g$ is the bandgap of the semiconductor, $E^{e}$ is the energy of free electrons on the hydrogen scale $(\sim 4.5 \mathrm{eV})$, and $X$ is the electronegativity of the semiconductor, which is $5.79 \mathrm{eV}$ and $5.81 \mathrm{eV}$ for $\mathrm{ZnO}$ QDs and $\mathrm{TiO}_{2} \mathrm{NPs}$, respectively. ${ }^{15}$ The $C B$ and $V B$ potentials calculated using Equations 4 and 5 are given in Table 1. 
The calculated $V B$ potential was $2.87 \mathrm{eV}$ for $\mathrm{ZnO}$ QDs, $2.95 \mathrm{eV}$ for $\mathrm{TiO}_{2} \mathrm{NPs}$, and $2.93 \mathrm{eV}$ for ZQT. The values are higher than the standard redox potentials of the oxidising agents such as ${ }^{\bullet} \mathrm{OH} / \mathrm{HO}^{-}(1.99 \mathrm{eV}), \mathrm{H}_{2} \mathrm{O}_{2}(1.77 \mathrm{eV})$, and $\mathrm{O}_{3}$ $(2.07 \mathrm{eV})$, suggesting that the synthesised nanocomposite may have stronger oxidation abilities. ${ }^{16}$ The calculated $C B$ potential for the $\mathrm{TiO}_{2} \mathrm{NPs}$ was more positive compared to $\mathrm{ZnO}$ QDs due to the QCE. The QCE shifts the $C B$ to more negative potentials (on the NHE scale) with decreasing particle size, leading to the generation of species with higher oxidation ability.
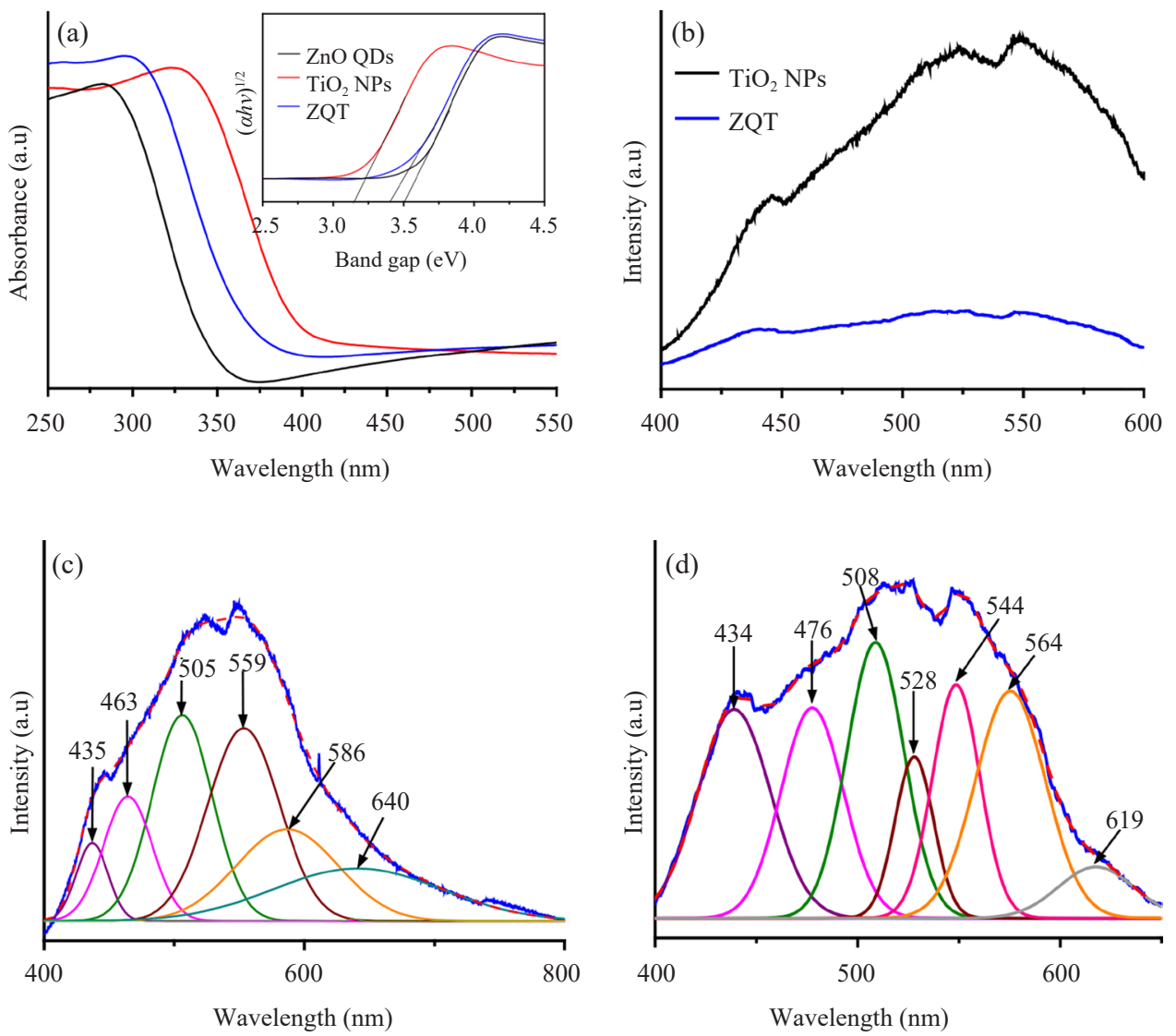

Figure 3: (a) UV-vis diffuse reflectance spectra with estimated energy gap (inset), (b) photoluminescence (PL) spectra of $\mathrm{TiO}_{2}$ NPs and ZQT, (c) deconvoluted PL spectrum of $\mathrm{TiO}_{2}$ NPs, and (d) deconvoluted PL spectrum of ZQT. 
Table 1: The $V B$ and $C B$ potentials of the $\mathrm{ZnO}$ QDs, $\mathrm{m}-\mathrm{TiO}_{2}$ and ZQT.

\begin{tabular}{lccc}
\hline Samples & Band gap $E g(\mathrm{eV})$ & $V B$ edge $(\mathrm{eV})$ & $C B$ edge $(\mathrm{eV})$ \\
\hline $\mathrm{ZnO}$ QDs & 3.50 & 2.87 & -0.46 \\
$\mathrm{TiO}_{2}$ NPs & 3.10 & 2.95 & -0.15 \\
$\mathrm{ZQT}$ & 3.24 & 2.93 & -0.30 \\
\hline
\end{tabular}

\subsection{Photoluminescence Study}

The PL intensity of ZQT was observed to be lower compared to $\mathrm{TiO}_{2} \mathrm{NPs}_{\text {(Figure }}$ $3[\mathrm{~b}]$ ), indicating that the separation of photogenerated $\mathrm{e}^{-} / \mathrm{h}^{+}$pairs in ZQT is better than $\mathrm{TiO}_{2}$ NPs. ${ }^{17}$ Four Gaussian bands were formed when the broad band of $\mathrm{TiO}_{2}$ NPs was deconvoluted (Figure 3[c]). The first Gaussian band at $435 \mathrm{~nm}$ is attributed to the self-trapped excitons of $\mathrm{TiO}_{6}$ octahedra. ${ }^{18}$ The Gaussian band at $463 \mathrm{~nm}$ indicate the shallow trap due to the $\mathrm{Ti}^{3+}$ states just below the conduction band, whereas the band at $505 \mathrm{~nm}$ is due to the deep trap state associated with the single electron trapped oxygen vacancy. ${ }^{19}$ The transitions of electrons from the conduction band edge to deep trap holes related to oxygen vacancies is indicated by the presence of a Gaussian band at $559 \mathrm{~nm} .{ }^{19}$ The intrinsic defects in the $\mathrm{TiO}_{2}$ NPs framework may have given rise to the Gaussian band at $586 \mathrm{~nm}$. The Gaussian band at $640 \mathrm{~nm}$ is associated with the surface oxygen of hydroxyl species which can create an acceptor level above the $V B$ of the $\mathrm{TiO}_{2} \mathrm{NPs}^{20}$

The ZQT's PL spectrum deconvolution resulted in six Gaussian bands indicating the formation of new defects sites (Figure 3[d]). The strong QCE of the ZnO QDs is indicated by a Gaussian band at $434 \mathrm{~nm} \cdot{ }^{21}$ The Gaussian band at $476 \mathrm{~nm}$ is attributed to the shallow trap assigned to the $\mathrm{Ti}^{3+}$ states just below the conduction band. ${ }^{19}$ The Gaussian band at $508 \mathrm{~nm}$ is due to the non-radiative electron capture from the $C B$ by a singly charged oxygen $\left(\mathrm{V}_{\mathrm{O}}^{+}\right)$vacancy leading to an unstable state that recombines with photogenerated holes in the $V B .{ }^{22}$ The transition of electrons from $C B$ to an oxygen antisite, $\mathrm{O}_{\mathrm{Zn}}$ is shown by the Gaussian band at $528 \mathrm{~nm} .{ }^{23}$ The Gaussian band at $544 \mathrm{~nm}$ is proposed to be due to the $C B \rightarrow \mathrm{O}_{i}$ transition by. ${ }^{24,25}$ The deeply trapped double-charged oxygen $\left(\mathrm{V}_{\mathrm{O}}{ }^{++}\right)$vacancy state, which undergoes recombination with a $C B$ electron, gives rise to the Gaussian band at $564 \mathrm{~nm}$ and $619 \mathrm{~nm} \cdot{ }^{17}$

\subsection{Photooxidation of Tetracycline}

The photocatalytic potential of the ZQT was tested in the photooxidation of tetracycline (TC) in an aqueous solution under fluorescent light irradiation. The photooxidation profiles are presented in Figure 4(a). Without ZQT, TC was not 
degraded, indicating TC's sufficient stability and the negligible self-photolysis effect. Both $\mathrm{TiO}_{2}$ NPs and $\mathrm{ZnO}$ QDs were able to photooxidise $32.4 \%$ and $68.8 \%$ of TC, respectively. The photooxidation of TC significantly enhanced up to $98.0 \%$ when ZQT was used. The higher photocatalytic activity of ZQT is attributed to the QCE of the $\mathrm{ZnO}$ QDs, besides the presence of various types of defects related to $\mathrm{TiO}_{2} \mathrm{NPs}$. The QCE will produce photogenerated $\mathrm{e}^{-} / \mathrm{h}^{+}$pairs that are highly energetic and capable of photoxidising pollutants. The TC photooxidation rate constant, $k$ was obtained based on the apparent first-order rate equation (Equation 6). The results are presented in Figure 4(b).

$$
-\ln \left(C / C_{0}\right)=k t
$$
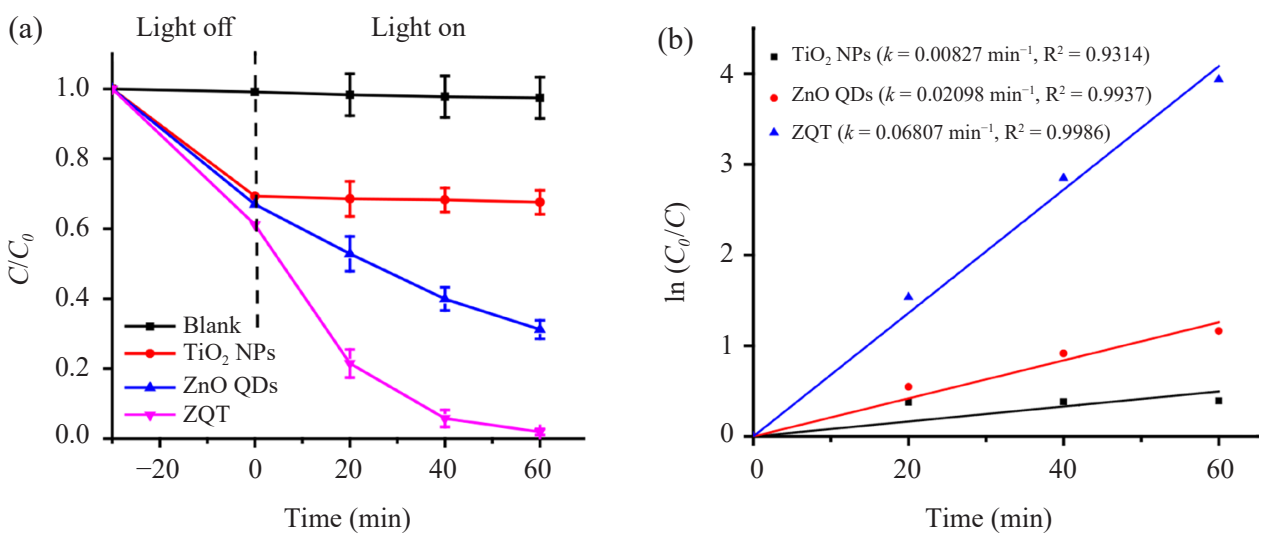

Figure 4: (a) Photocatalytic degradation of TC using different catalysts, and (b) kinetic curve to evaluating the rate constants $(k)$.

Scavenging tests were conducted to identify the active species responsible for the photodegradation of TC. Ascorbic acid (AA), triethanolamine (TEA), and isopropanol (IPA) were used as a scavenging agent for $\mathrm{O}_{2}{ }^{\bullet}, \mathrm{h}^{+}$, and ${ }^{\bullet} \mathrm{OH}$, respectively. ${ }^{26-29}$ From the TC removal profile shown in Figure 5(a), the TC removal dropped to $21.87 \%$ and $60.94 \%$ when AA and IPA were added, whereas, in the presence of TEA, $73.27 \%$ of TC was removed, respectively. Hence, the major species involved in the photodegradation process was $\mathrm{O}_{2}{ }^{\bullet-}$ followed by ${ }^{\bullet} \mathrm{OH}$ and $\mathrm{h}^{+}$. Based on the scavenging test data, the flow of photogenerated $\mathrm{e}^{-} / \mathrm{h}^{+}$is proposed. From Table 1 , the $\mathrm{TiO}_{2} \mathrm{NPs}$ has the $C B$ and $V B$ potential of $2.95 \mathrm{eV}$ and $-0.15 \mathrm{eV}$, respectively. The $C B$ and $V B$ potential of $\mathrm{ZnO}$ QDs is $2.87 \mathrm{eV}$ and $-0.46 \mathrm{eV}$, respectively. When irradiated, electrons from the $V B$ band of both semiconductors will be excited to their respective $C B$. Since the reduction potential of oxygen $\left(\mathrm{E}^{0}\left(\mathrm{O}_{2} / \mathrm{O}_{2}{ }^{--}\right)=-0.33 \mathrm{eV} / \mathrm{NHE}\right)$ is more negative than the $C B$ potential of $\mathrm{TiO}_{2} \mathrm{NPs}$, it will not have enough reduction abilities to 

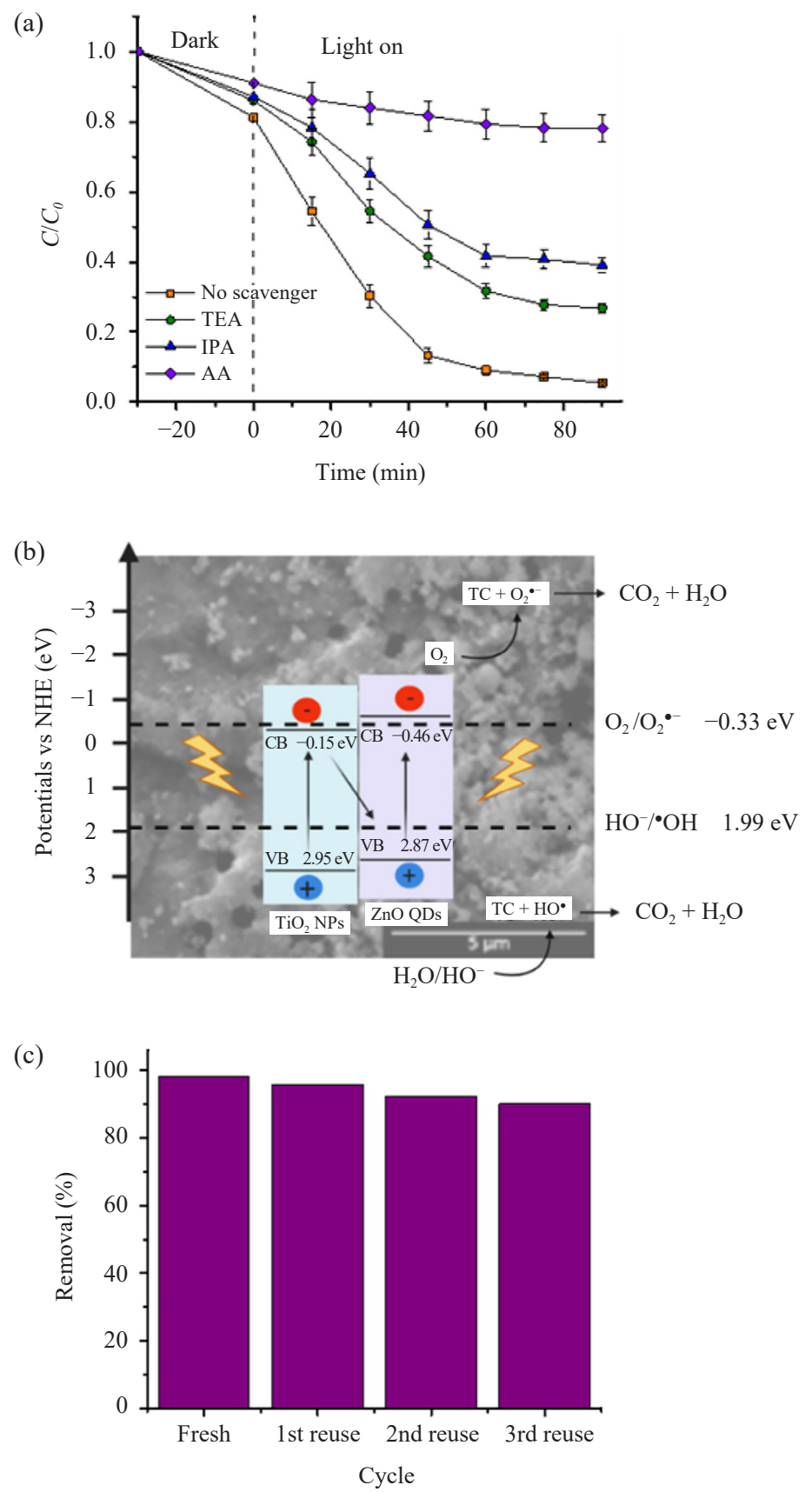

Figure 5: (a) Photocatalytic degradation of TC in the presence of different scavengers, (b) the flow of photogenerated electron/hole pairs, and (c) the reusability profile. 
generate $\mathrm{O}_{2}{ }^{\bullet-}$ species from dissolved $\mathrm{O}_{2}$. Hence, the Z-scheme heterojunction is proposed since the $C B$ potential of $\mathrm{ZnO}$ QDs can generate $\mathrm{O}_{2}{ }^{--}$species. The potential of the photogenerated holes in the $V B$ of $\mathrm{TiO}_{2} \mathrm{NPs}$ is positive enough to oxidise $\mathrm{OH}^{-}$to ${ }^{\bullet} \mathrm{OH}\left(\mathrm{E}^{0}\left(\mathrm{OH}-/^{\bullet} \mathrm{OH}=+1.99 \mathrm{eV}\right)\right.$ and $\mathrm{H}_{2} \mathrm{O}$ to ${ }^{\bullet} \mathrm{OH}\left(\mathrm{E}^{0}\left(\mathrm{H}_{2} \mathrm{O} /{ }^{\bullet} \mathrm{OH}\right.\right.$ $=+2.34 \mathrm{eV}$ ). The photogenerated electrons in the $C B$ of $\mathrm{TiO}_{2} \mathrm{NPs}$ will migrate to the $V B$ band of $\mathrm{ZnO}$ QDs to recombine with the photogenerated holes. Based on the Z-scheme, the photogenerated electrons will accumulate in the $V B$ of $\mathrm{ZnO}$ QDs, whereas the holes will remain in the $C B$ band of the $\mathrm{TiO}_{2} \mathrm{NPs}$. Hence, the pairs can be separated effectively. The generated $\mathrm{O}_{2}{ }^{\bullet-}$ and ${ }^{\circ} \mathrm{OH}$ radicals can effectively degrade the TC into $\mathrm{CO}_{2}$ and $\mathrm{H}_{2} \mathrm{O}$. The flow of photogenerated $\mathrm{e}^{-} / \mathrm{h}^{+}$pairs, according to the Z-scheme, is shown in Figure 5(b). Figure 5(c) shows the reusability profile of ZQT in TC photodegradation. The ZQT experienced $\sim 8 \%$ loss in its photocatalytic activity at the end of four cycles. The small degree of loss indicates that ZQT has good stability.

\section{CONCLUSION}

In this study, a highly porous ZQT nanocomposite was successfully prepared by embedding $\mathrm{ZnO}$ QDs within the matrix of anatase $\mathrm{TiO}_{2}$ through sol-gel assisted hydrothermal method. The embedment of $\mathrm{ZnO}$ QDs introduced its quantum confinement effect (QCE) to the anatase $\mathrm{TiO}_{2}$. The QCE and the defects related to $\mathrm{TiO}_{2}$ and oxygen vacancies significantly enhanced the photodegradation of tetracycline (TC) compared to $\mathrm{TiO}_{2} \mathrm{NPs}$. The QCE will produce photogenerated electron/hole pairs with higher energy. At the same time, the presence of $\mathrm{Z}$-scheme heterojunction suppressed the recombination rate of the photogenerated $\mathrm{e}^{-} / \mathrm{h}^{+}$pairs. In ZQT's presence, $98 \%$ of TC was removed, whereas $32.4 \%$ and $68.8 \%$ of $\mathrm{TC}$ was removed when $\mathrm{TiO}_{2} \mathrm{NPs}$ and $\mathrm{ZnO}$ QDs were used.

\section{ACKNOWLEDGEMENTS}

This research was supported by Universiti Sains Malaysia Research University Grant (RUI) (1001/PKIMIA/8011083). Usman Saidu acknowledges Sule Lamido University Kafin Hausa, Nigeria, for the PhD sponsorship awarded to him through Tertiary Education Trust Fund (TETFund). 


\section{REFERENCES}

1. Ahmed, S. N. \& Haider, W. (2018). Heterogeneous photocatalysis and its potential applications in water and wastewater treatment: A review. Nanotechnology, 29(34), 342001. https://doi.org/10.1088/1361-6528/aac6ea

2. Li, D. \& Shi, W. (2016). Recent developments in visible light photocatalytic degradation of antibiotics. Chinese J. Catal., 37(6), 792-799. https://doi.org/ 10.1016/s1872-2067(15)61054-3

3. Yang, J. et al. (2018). $\mathrm{BiVO}_{4}$ quantum tubes loaded on reduced graphene oxide aerogel as efficient photocatalyst for gaseous formaldehyde degradation. Carbon, 138, 118-124. https://doi.org/10.1016/j.carbon.2018.06.003

4. Miodyńska, M. et al. (2020). Urchin like $\mathrm{TiO}_{2}$ structures decorated with lanthanide doped $\mathrm{Bi}_{2} \mathrm{~S}_{3}$ quantum dots to boost hydrogen photogeneration performance. Appl. Catal. B., 272, 118962. https://doi.org/10.1016/j.apcatb.2020 .118962

5. Zhang, D. et al. (2019). The in situ ligand exchange linker assisted assembly of oil soluble CdSe quantum dots to $\mathrm{TiO}_{2}$ films. Appl. Surf. Sci., 475, 813-819. https://doi.org/10.1016/j.apsusc.2018.12.289

6. Chen, S., Li, C. \& Hou, Z. (2019). A novel in situ synthesis of $\mathrm{TiO}_{2} / \mathrm{CdS}$ heterojunction for improving photoelectrochemical water splitting. Int. $J$. Hydrog. Energ., 44(47), 25473-25485. https://doi.org/10.1016/j.ijhydene.2019 .08 .049

7. Basheva, E. S. et al. (2020). In situ growth of CdS quantum dots on phosphorus doped carbon nitride hollow tubes as active 0D/1D heterostructures for photocatalytic hydrogen evolution. J. Colloid Interface Sci., 577, 1-11. https://doi.org/10.1016/j.jcis.2020.05.053

8. Ghows, N. \& Entezari, M. H. (2012). Sono synthesis of core shell nanocrystal $\left(\mathrm{CdS} / \mathrm{TiO}_{2}\right)$ without surfactant. Ultraso. Sonochem., 19(5), 1070-1078. https://doi.org/10.1016/j.ultsonch.2012.01.009

9. Mahjoub, M. A. et al. (2016). Synthesis and study of stable and size controlled $\mathrm{ZnO} \mathrm{SiO} 2$ quantum dots: Application as a humidity sensor. J. Phys. Chem. C., 120(21), 11652-11662. https://doi.org/10.1021/acs.jpcc.6b00135

10. Yin, Q. et al. (2016). Preparation of highly crystalline mesoporous $\mathrm{TiO}_{2}$ by solgel method combined with two-step calcining process. J. Expl. Nanosci., 11(14), 1127-1137. https://doi.org/10.1080/17458080.2016.1189097

11. Qiao, B. et al. (2016). Synthesis of $\mathrm{ZnO}$ quantum dots and their agglomeration mechanisms along with emission spectra based on ageing time and temperature. Chin. Phys. B., 25(9), 098102. https://doi.org/10.1088/1674-1056/25/9/098102

12. Iqbal, A. et al. (2021). Floating $\mathrm{ZnO}$ QDs-modified $\mathrm{TiO}_{2} / \mathrm{LLDPE}$ hybrid polymer film for the effective photodegradation of tetracycline under fluorescent light irradiation: Synthesis and characterisation. Molecules, 26, 2509. https://doi.org/ $10.3390 /$ molecules 26092509 
13. Chen, Q. et al. (2018). Ultrafine $\mathrm{ZnO}$ quantum dot modified $\mathrm{TiO}_{2}$ composite photocatalysts: The role of the quantum size effect in heterojunction enhanced photocatalytic hydrogen evolution. Catal. Sci. Technol., 8(5), 1296-1303. https://doi.org/10.1039/c7cy02310c

14. Muniandy, S. S. et al. (2017). Green synthesis of mesoporous anatase $\mathrm{TiO}_{2}$ nanoparticles and their photocatalytic activities. RSC Adv., 7(76), 48083-48094. https://doi.org/10.1039/C7RA08187A

15. Jiang, Y. et al. (2018). Facile in-situ solvothermal method to synthesise double shell $\mathrm{ZnIn}_{2} \mathrm{~S}_{4}$ nanosheets/ $\mathrm{TiO}_{2}$ hollow nanosphere with enhanced photocatalytic activities. Ceram. Int., 44(6), 6115-6126. https://doi.org/10.1016/j.ceramint.2017 .12 .244

16. Li, W. et al. (2018). Photocatalytic degradation of tetracycline hydrochloride via a $\mathrm{CdS}-\mathrm{TiO}_{2}$ heterostructure composite under visible light irradiation. Nanomaterials, 8(6), 415. https://doi.org/10.3390/nano8060415

17. Imam, S. S., Adnan, R. \& Kaus, N. H. M. (2018). Influence of yttrium doping on the photocatalytic activity of bismuth oxybromide for ciprofloxacin degradation using indoor fluorescent light illumination. Res. Chem. Intermed., 44(9), 53575376. https://doi.org/10.1007/s11164-018-3427-8

18. Paul, K. K. \& Giri, P. K. (2017). Role of surface plasmons and hot electrons on the multi step photocatalytic decay by defect enriched $\mathrm{Ag} @ \mathrm{TiO}_{2}$ nanorods under visible light. J. Chem. C., 121(36), 20016-20030. https://doi.org/10.1021/acs .jpcc. $7 \mathrm{~b} 05328$

19. Khan, H. \& Swati, I. K. (2016). $\mathrm{Fe}^{3+}$-doped anatase $\mathrm{TiO}_{2}$ with d-d transition, oxygen vacancies and $\mathrm{Ti}^{3+}$ centers: Synthesis, characterisation, UV-vis photocatalytic and mechanistic studies. Ind. Eng. Chem. Res., 55(23), 6619-6633. https://doi.org/10.1021/acs.iecr.6b01104

20. Santara, B. et al. (2013). Evidence of oxygen vacancy induced room temperature ferromagnetism in solvothermally synthesised undoped $\mathrm{TiO}_{2}$ nanoribbons. Nanoscale, 5(12), 5476. https://doi.org/10.1039/c3nr00799e

21. Ghorai, A. et al. (2016). Highly luminescent $\mathrm{WS}_{2}$ quantum dots/ZnO heterojunctions for light emitting devices. ACS Appl. Mater. Interfaces, 9(1), 558-565. https://doi.org/10.1021/acsami.6b12859

22. Vanheusden, K. et al. (1996). Mechanisms behind green photoluminescence in ZnO phosphor powders. J. Appl. Phys., 79(10), 7983-7990. https://doi.org/10 $.1063 / 1.362349$

23. Pal, M. et al. (2012). Effects of crystallisation and dopant concentration on the emission behavior of $\mathrm{TiO}_{2}$ : Eu nanophosphors. Nanoscale Res. Lett., 7(1), 1. https://doi.org/10.1186/1556-276x-7-1

24. Ahn, C. H. et al. (2009). A comparative analysis of deep level emission in $\mathrm{ZnO}$ layers deposited by various methods. J. Appl. Phys., 105(1), 013502. https://doi.org/10.1063/1.3054175

25. Cao, B., Cai, W. \& Zeng, H. (2006). Temperature-dependent shifts of three emission bands for $\mathrm{ZnO}$ nanoneedle arrays. Appl. Phys. Lett., 88(16), 161101. https://doi.org/10.1063/1.2195694 
26. Nasseh, N. et al. (2020). Enhanced photocatalytic degradation of tetracycline from aqueous solution by a novel magnetically separable $\mathrm{FeNi}_{3} / \mathrm{SiO}_{2} / \mathrm{ZnO}$ nanocomposite under simulated sunlight: Efficiency, stability and kinetic studies. J. Mol. Liq., 301, 112434. https://doi.org/10.1016/j.molliq.2019.112434

27. Zhang, H. et al. (2018). Enhanced photocatalytic degradation of ciprofloxacin using novel C-dot@Nitrogen Deficient g- $_{3} \mathrm{~N}_{4}$ : Synergistic effect of nitrogen defects and C-dots. Appl. Surf. Sci., 465, 450-458. https://doi.org/10.1016/j. apsusc.2018.09.183

28. Shi, W., Guo, F. \& Yuan, S. (2017). In situ synthesis of Z-scheme $\mathrm{Ag}_{3} \mathrm{PO}_{4} / \mathrm{CuBi}_{2} \mathrm{O}_{4}$ photocatalysts and enhanced photocatalytic performance for the degradation of tetracycline under visible light irradiation. Appl. Catal. B., 209, 720-728. https://doi.org/10.1016/j.apcatb.2017.03.048

29. Yan, Q. et al. (2016). Efficient photocatalytic degradation of tetracycline hydrochloride by $\mathrm{Ag}_{3} \mathrm{PO}_{4}$ under visible-light irradiation. Environ. Sci. Pollut. Res., 23(14), 14422-14430. https:// doi:10.1007/s11356-016-6588-2 